\title{
Antropología
}

\section{La salud y las creencias del pueblo gitano en Europa: revisión bibliográfica}

Health and beliefs of the Roma in Europe: a review

\section{Saúdle e crenças dos ciganos na Europa: uma literature revisão}

\author{
María Gemma Tuset-Garijoㅜㄹ Alba Roca-Biosca²; Pilar Alamillo Pérez- Grueso; \\ María Antonia Martorell-Poveda ${ }^{2}$
}

${ }^{1}$ Enfermera asistencial Servicio de Medicina Intensiva. Hospital Universitari de Tarragona Joan XXIII.
${ }^{2}$ Profesora Departamento de Enfermería Universidad Rovira i Virgili de Tarragona.

Cómo citar este artículo en edición digital: Tuset Garijo, Ma .G; Roca Biosca, A; Alamillo Pérez Grueso, P; Martorell Poveda $M^{a}$.A.(2012) La salud y las creencias del pueblo gitano en Europa: revisión bibliográfica.16, 34. Disponible en: $<$ http://dx.doi. org/10.7184/cuid.2012.34.09>

Correspondencia: Ma.G. Tuset-Garijo. Calle Mallorca 25, 2M.n C.P. 43005. Tarragona. E-mail: gtuset2010@gmail.com Recibido 11/06/2012/ Aceptado: 10/09/2012

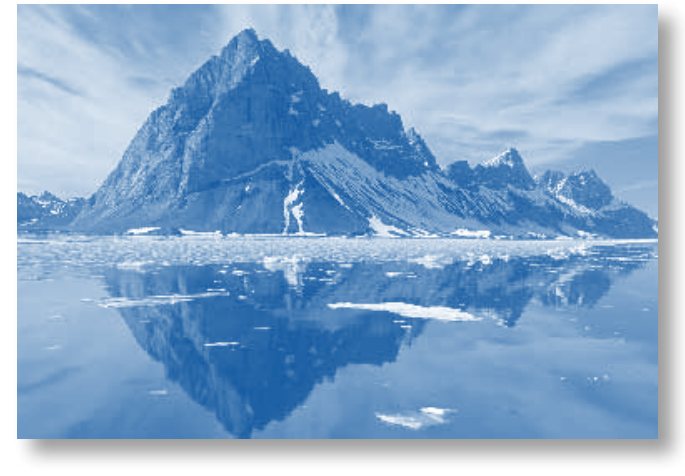

ABSTRACT

Introduction: The Roma community is the largest ethnic minority in Spain, Central and Eastern Europe. As an ethnic group is characterized by a series of cultural elements that shape its ethnic content, its own cultural baggage that distinguishes it from other groups with which it comes into contact. Provide health care services that generally do not address cultural considerations.

Aim: to have a greater understanding of the health of the European Roma and his personal view on the concept of health and illness in order to provide an effective tool to help nurses to provide proper care from a multicultural perspective.

Method: In the first search was conducted a review of the literature published over the past 30 years in the databases PubMed, Cuiden, Scopus, Cinalh, Index, Spanish physician, ISOC-CSIC and multicercador URV. In a second search used the metasearch "google. com" and "metacrawler.com".

Results: We found 102 articles related to the subject to revision of which 13 were considered crucial for providing useful information relevant to the review study. The topics covered were referring to genetic diseases, to sociology and anthropology of health care, child health and transmissible diseases.

Keywords: gypsy, illness and cultures.

\section{RESUMO}

Introdução: A comunidade cigana é a maior minoria étnica na Espanha, Europa Central e Oriental. Como um grupo étnico é caracterizado por uma série de elementos cul- 
turais que moldelam o seu conteúdo étnico, a sua própria bagagem cultural que o distingue de outros grupos com os quais entra em contato. Os serviços de saúde prestam cuidados que geralmente não abordam aspectos culturais.

O objetivo desta revisão é ter um maior entendimento da saúde da Roma Europeu e sua visão pessoal sobre o conceito de saúde e doença, a fim de proporcionar uma ferramenta eficaz para ajudar os enfermeiros para prestar assistência adequada a partir de uma perspectiva multicultural.

Método: Na primeira pesquisa foi realizada uma revisão da literatura publicada nos últimos 30 anos nas bases de dados PubMed ,Cuiden, Scopus, Cinalh,, Index, Médico Espanhol, ISOC-CSIC e multicercador-URV. Em uma segunda pesquisa utilizou a metabusca "google.com" e "metacrawler.com".

Resultados: Foram encontrados 102 artigos relacionados com o objecto de revisão, dos quais 13 foram considerados cruciais para proporcionar informações úteis e relevantes para o estudo de revisão. Os temas abordados foram referentes a doenças genéticas, a sociologia ea antropologia dos cuidados de saúde, saúde da criança e das doenças transmissíveis.

Palavras-chave: cigano, doença e cultura.

\section{RESUMEN}

Introducción: La comunidad gitana constituye la minoría étnica más importante en España, Europa central y del Este. Como grupo étnico, se caracteriza por una serie de elementos culturales comunes que configuran su contenido étnico. Los servicios sanitarios proporcionan cuidados que, en general, no atienden a consideraciones culturales. Objetivo: tener una mayor comprensión sobre el estado de salud de la etnia gitana europea y su particular visión sobre el concepto de salud-enfermedad con el objeto de proporcionar una herramienta que ayude a los profesionales de enfermería a proporcionar unos cuidados desde una perspectiva multicultural.

Método: En una primera búsqueda se realizó una revisión de la bibliografía publicada en los últimos 30 años en las bases de datos Pubmed, Cuiden, Scopus, Cinalh,, Índice medico español, ISOC-CSIC y multicercador URV. En una segunda búsqueda se utilizaron los metabuscadores "google.com" y "metacrawler.com".

Resultados y discusión: Se encontraron 102 artículos relacionados con el tema a revisión de los cuales 13 fueron considerados clave por aportar datos de interés relevantes en el estudio de revisión. Los temas más tratados fueron los que hacían referencia a las afecciones genéticas, a la Sociología y antropología médica de la salud, a la salud infantil y a las enfermedades transmisibles.

Palabras clave: gitano, enfermedad y cultura.

\section{INTRODUCCIÓN}

"Está claro que no somos todos iguales, pero seguramente no habríamos llegado hasta aquí los unos sin los otros". Anónimo

El desarrollo de los procesos migratorios en Europa durante los últimos años ha producido una demanda de salud por parte de una población cada vez más multicultural. Los servicios sanitarios proporcionan cuidados que, en general, no atienden a consideraciones culturales. Se hace necesario un cambio de las teorías y la práctica enfermera que responda a estas nuevas necesidades. Madeleine Leininger (1991) abre un nuevo camino hacia la diversidad y universalidad de los cuidados entendiendo por cultura "los valores, las creencias, las normas y los modos de vivir compar- 
tidos, aprendidos y transmitidos que guían los pensamientos, las decisiones y las acciones de un grupo de manera específica".

Los profesionales son cada vez más conscientes de la necesidad de comprender la variación cultural en las creencias y prácticas médicas (Casabona y Lillo, 2006). Señalan que la medicina transcultural, la medicina de las minorías y los emigrantes deben recibir mucha más atención. Cuanto más sabemos acerca de cómo la salud y la enfermedad son administradas en las diferentes culturas, más fácil es reconocer la vinculación de la "Cultura" en nuestras propias creencias y prácticas médicas (Pavlič, Zelko, Kersnik y Lolić, 2011).Teniendo en cuenta que en España, Europa central y Europa del Este, los gitanos constituyen la minoría étnica más importante, es necesario tener una visión cultural de esta comunidad para un mayor entendimiento de sus conductas y relaciones con el sistema sanitario.

La marginación del pueblo gitano es el resultado de un largo proceso histórico. Tiene un remoto origen oriental, en el noroeste de la India, desde donde emigró al Mediterráneo oriental y a Centroeuropa. Su origen siempre fue objeto de disputa y especulación. A finales del siglo XVIII hubo estudiosos europeos que se percataron de que el romano (idioma gitano) era de origen indio. Otros especialistas rastrearon la ruta que los Gitanos habían seguido a través del norte de la India, Armenia, hasta Europa, pasando por el imperio de $\mathrm{Bi}$ zancio. Numerosas publicaciones hacen referencia en la literatura medio-oriental al origen indio de los Gitanos (Kenrik, 1995).

Entender el hoy y el ahora de la comunidad gitana implica conocer no sólo su historia, sino las formas en las que históricamente se han conformado las relaciones entre mayoría y minoría; siendo el vector más reseñable la profunda desigualdad que ha regido desde su inicio las relaciones entre sociedad gitana $y$ paya (Otegui, 2005).

La historia del pueblo gitano en España ha sido estudiada espléndidamente por Teresa San Román (1986) quien nos permite conocer desde su llegada y asentamiento en este país, hasta los diferentes aspectos sociales y culturales que les caracteriza.

Un ejemplo de la marginación sufrida por esta minoría étnica es que, pese a las numerosas publicaciones consagradas a la masacre de los judíos, muy poco se ha escrito sobre el holocausto de los gitanos (la original población gitana de Bohemia fue exterminada durante la Segunda Guerra Mundial y los actuales pobladores son en su mayoría descendientes de inmigrantes procedentes de Eslovaquia y Bulgaria) (Seeman y SisKová, 2005). Éstos no han dejado testimonio escrito de lo sucedido, pero han transmitido oralmente la historia a sus hijos, o han tratado de olvidar esos años sangrientos.

La comunidad gitana, como grupo étnico, se caracteriza por una serie de elementos culturales comunes que configuran su bagaje cultural propio que lo distingue de otros grupos con los que entra en contacto. La idea de un origen común, la tradición nómada, la lengua, la valoración de la edad y la experiencia como principios ordenadores de status, el respeto a los muertos y la creencia en una intervención de éstos en la vida de sus descendientes, son algunos de los rasgos que se encuentran en las distintas colectividades que se reconocen como gitanas y que constituye parte de este contenido étnico necesario para la supervivencia del grupo como unidad. Los problemas de marginación, aculturación e identidad étnica de los gitanos, no han impedido la transformación social de esta comunidad. 
Los gitanos, como el resto de los ciudadanos, están sujetos a las leyes y a la ordenación de la justicia. Sin embargo, "la ley gitana", como llaman a su conjunto de normas tradicionales, discurren paralelas al ordenamiento jurídico del país. Esta duplicidad sitúa al individuo en un terreno conflictivo en el que normas contradictorias que se asientan sobre los valores $y$ condiciones muy diferentes, entran en juego forzando a los gitanos a realizar opciones que con frecuencia implican transgresiones en el código opuesto (San Roman, 1986).

En lo que a la sanidad se refiere, pese a presentar unas necesidades de salud muy específicas, llama la atención la escasez de trabajos publicados al respecto y el desinterés de los profesionales sanitarios (Otegui, 2005; Cabedo et al, 2000; Casals, Pila, Langohr, Millet y Caylà, 2012). En el ámbito de la investigación de la medicina social es bien conocido lo dificultoso que resulta comparar dos poblaciones, sobre todo si se trata de hacerlo por su condición étnica. No existen registros oficiales para estudiar y establecer comparaciones entre la población gitana y no gitana (Sastre, Miranda, Muñoz y Galdó, 2000).

En España se ha realizado estudios que muestran las desigualdades socioeconómicas en salud, de cuya repercusión en el pueblo gitano son conscientes las autoridades (en la VI legislatura española en el Congreso de los Diputados se presentaron más de medio centenar de iniciativas sobre la problemática del pueblo gitano).

El objetivo de esta revisión es tener una mayor comprensión sobre el estado de salud de la etnia gitana europea y su particular visión sobre el concepto de salud-enfermedad con el objeto de proporcionar una herramienta eficaz que ayude a los profesionales de enfermería a proporcionar unos cuidados desde una perspectiva multicultural.

\section{METODOLOGÍA DE LA BUSQUEDA}

Para poder evaluar la investigación publicada sobre la salud de la etnia gitana en Europa ha sido necesario llevar a cabo una extensa revisión de la literatura publicada durante los últimos 30 años. Se trata de una revisión descriptiva que intenta proporcionar una puesta al día sobre un tema de interés sanitario analizado desde el punto de vista de la interculturalidad.

La primera búsqueda se realizó utilizando las palabras clave en español: gitano, enfermedad y cultura y las palabras clave en ingles: gipsy, roma, disease e illness.

Se consideraron como criterios de inclusión todos los artículos publicados en lengua inglesa o española a nivel mundial en los últimos 30 años sin hacer distinción entre cualitativos y/o cuantitativos.

Se excluyeron aquellos artículos que hacían referencia a la población gitana no europea.

Las bases de datos a las que se accedió fueron: Pubmed, Cinalh, Scopus, Índice Medico Español, Cuiden, ISOC-CSIC, Scielo, y la plataforma electrónica Elsevier i JSTOR. También se utilizó el buscador de la Universitat Rovira i Virgili (multicercador URV).

Se consideró que podía haber una información no publicada por métodos tradicionales o elaborados por organismos o asociaciones no científicas. Por ello se realizó una segunda búsqueda en Internet mediante los metabuscadores "google.com" y "metacrawler. com", acotando a" idioma español" y utilizando la palabra clave "gitano" y "enfermedad".

Como resultado de esta búsqueda alternativa se encontró una publicación bimensual de la Fundación del Secretariado Gitano que aportó a la revisión información de interés. Ésta es una entidad social sin ánimo de lucro que presta servicios para el desarrollo de la co- 
munidad gitana en todo el territorio Español y en el ámbito europeo. De igual modo se hallaron publicaciones de ámbito gubernamental, nacional y autonómico que fueron consideradas de interés por aportar información fidedigna y actualizada sobre el tema en revisión.

También fueron consultados manuales publicados en el campo de las Ciencias Sociales y Antropología Médica con el objeto de hallar un marco conceptual de referencia.

El total de los artículos incluidos en la revisión bibliográfica se clasificaron por un lado según el país de origen de la publicación y el idioma de edición de la revista. Por otro lado se hizo una segunda clasificación según el origen de los sujetos de estudio y los temas de salud tratados. Tras la búsqueda, todos los documentos fueron leídos y evaluados, recopilando aportaciones y conclusiones sobre el tema que nos ocupa.

\section{RESULTADOS}

Como resultado global se obtuvieron un total de 102 citas bibliográficas publicadas en revistas nacionales el $68 \%$ de los casos y extranjeras el 32\% restante. En estas últimas el idioma del artículo era el inglés. La estrategia de búsqueda se muestra en la figura 1 .

En la figura 2 se muestra un resumen de los artículos escogidos en base a los criterios de inclusión y exclusión previamente descritos.

Como resultado de la búsqueda utilizando "Google.com" fueron hallados aproximadamente 13.800.000 resultados. El meta buscador "metacrawler.com" no permite cuantificar el número de resultados hallados.

El 90\% de los estudios revisados eran de tipo analítico y/o descriptivo y solo el 10\% eran trabajos de revisión. En el caso de los estudios todos tenían diseño observacional de los que $60 \%$ eran descriptivos y el resto analíticos.
Los temas de salud tratados se clasificaron en 6 apartados: sociología y antropología médica de la salud, reproducción y mujer, salud infantil e inmunización, enfermedades transmisibles (incluidos SIDA y HIV), afecciones genéticas y otros.

La distribución de los estudios revisados a texto completo según el tema de salud tratado $(n=62)$ se muestra en la tabla 1.

La distribución de los estudios revisados a texto completo $(n=62)$ según país de edición de la revista se muestra en la tabla 2.

Los datos referentes al país de origen de los sujetos participantes de los estudios se muestran en la tabla 3.

Tras el examen de la literatura, se exponen a continuación todos aquellos conceptos y conocimientos publicados en la actualidad, necesarios para entender el estado de salud de la comunidad gitana y alcanzar un nuevo enfoque enfermero que permita el cuidado desde una perspectiva cultural.

\section{DISCUSIÓN}

Los procesos migratorios en Europa han originado una composición social cada vez más multicultural, siendo la minoría étnica gitana la más numerosa.

Todos los estudios coinciden en la dificultad que supone establecer con precisión el número exacto de gitanos en la Unión Europea. Las estimaciones más recientes sitúa la cifra alrededor de los 10 millones según el informe del 2008 del Parlamento Europeo. En España, la comunidad gitana oscila entre $600.000 \mathrm{y}$ 1.000.000 de individuos (Otegui, 2005; Casals e al, 2012; García y Alda, 2007).

Para poder comprender las necesidades de este colectivo es necesario conocer su realidad, a través del estudio de la historia, de los diferentes asentamientos que ha realizado y de las 
formas en las que históricamente se han conformado las relaciones de esta minoría con la población mayoritaria con la que le ha tocado coexistir. Allá donde han migrado, conservan los elementos estructurales imprescindibles para su supervivencia y reproducción como grupo. Es reseñable la profunda desigualdad que ha regido desde su inicio en las relaciones entre sociedad gitana y paya, pero han aprendido a convivir mediante formas culturales adaptativas aunque no transformativas.

La mayoría de los artículos revisados refieren una menor esperanza de vida en esta comunidad (alrededor de 10 años), así como una tasas de fertilidad y de mortalidad infantil (alrededor de 4 veces mayor que la población no gitana), siendo por tanto una población con una media de edad más joven (Pavlič et al, 2011; Cabedo et al, 2000; Casals et al, 2012; García y Alda, 2007; Masseria, Mladovsky y Hernández-Quevedo, 2010).

Susana Ramírez Hita (2007) observó que la etnia gitana utiliza los mismos sistemas médicos (biomédico, tradicional, auto-atención y religioso) en contextos geográficos diferentes. Casi todas las investigaciones sobre la salud llevadas a cabo en este colectivo se centran en la medicina tradicional o en la biomédica. Por lo tanto, al no ser considerados todos los sistemas de salud a los que recurre la población gitana, no puede hacerse una interpretación completa del concepto salud-enfermedad.

David Lagunas (1999) en su artículo "Resolviendo la salud los gitanos catalanes" realiza un estudio antropológico muy interesante sobre la visión y conocimientos sobre la salud y la enfermedad, así como su gestión ante el aparato asistencial, de un grupo particular de gitanos en Cataluña-España. Llama la atención la creencia de los gitanos que la curación de las enfermedades es efectiva si se acude a

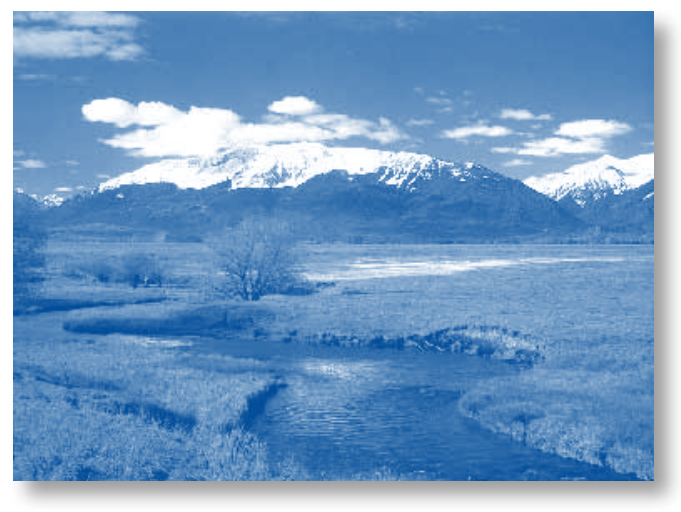

los médicos que cuestan más dinero, además de consumir los medicamentos más caros. Paradójicamente la mayoría de los artículos señalan que este colectivo presenta desigualdades sociales, económicas y sanitarias respecto al resto de la población así como dentro del propio colectivo (Pavlič e al, 2011; Casals et al, 2012; García y Alda, 2007; Masseria et al, 2010; Kelly et al, 2006; Cabedo et al. 2000) señalan que entre la población gitana observada en su estudio existe altos niveles de pobreza y altas tasas de desempleo o sin trabajo estable, siendo la mayoría pensionistas o parados sin subsidio. Jeffrey A Kelly et al (2006) también avalan la idea que en la mayoría de países los gitanos ya no son nómadas (estereotipada idea sobre los gitanos) pero por lo general viven en asentamientos que se diferencian de la mayoría de la comunidad y se caracterizan por la pobreza, el hacinamiento y viviendas deterioradas, con un saneamiento deficiente, y con pocos servicios de salud pública. Estos factores son determinantes del estado de bienestar $y$ calidad de vida de una población en un entorno determinado (Otegui, 2005; García y Alda, 2007; Kelly et al, 2006).

Para Rosario Otegui (2005) la comunidad gitana tiene una concepción particular de la representación salud/enfermedad, que, alejada de la perspectiva biomédica hegemónica, incorpora además de sus características biológi- 
cas individuales, sus rasgos biológicos sociales. En esto coincide con David Lagunas (1999), quien afirma que la enfermedad (su origen) se vincula a una transgresión del orden social que se traslada al cuerpo. En general, la buena salud del hombre se asocia con la fuerza y la virilidad, mientras que para la mujer se asocia a la fertilidad, a su estética (estar gorda y no delgada). La salud también se vincula a una extremada preocupación por la higiene corporal, especialmente para las mujeres. El pelo, por ejemplo, tiene un papel muy sobresaliente en la cultura gitana: un pelo largo, lustroso, abundante y cuidado es uno de los mayores atractivos de un hombre y una mujer gitana (Otegui, 2005). En el artículo "Health beliefs and practices among slovenian Roma and their response to febrile illnesse: a quantitative study" (Pavlič et al, 2011) se hace referencia al significado de salud para los gitanos y concluye que la salud es asociada al sueño profundo, la respiración normal, no tener fiebre y la ausencia de dolor.

El mismo artículo señala que los gitanos todavía mantienen algunas creencias antiguas, supersticiones y respuestas que ya no son comunes hoy en día pero que ellos han conservado, se cree que, como resultado de la estigmatización a la que han sido sometidos en Europa durante los siglos pasados. Una maldición, por ejemplo tiene el poder de crear la enfermedad en otra persona. En todo caso, se revela la necesidad de interpretar la enfermedad independientemente de los modelos médicos, de asignarle causas en términos no orgánicos y darle un sentido (Lagunas, 1999).

En cuanto a su gestión ante el aparato asistencial, Cabedo et al (2000), en su artículo "Cómo son y de qué padecen los gitanos", afirma que los miembros de esta étnia reconocen determinadas enfermedades como pro- pias de su cultura y por tanto son tratadas por sanadores propios con remedios tradicionales mientras que, para otras, que son consideradas producto de su contacto con el resto del mundo, requieren ayuda del sistema médico oficial. Existe consenso al afirmar que los gitanos son hipocondríacos y que tienen miedo de ir a la consulta médica ya que creen que si van, éste les diagnosticará alguna enfermedad. Prefieren estar en un estado de incerteza y acudir sólo en el caso de presentar síntomas agudos y diagnósticos y tratamiento inmediato. El médico es una figura bivalente: por un lado posee el saber para curar a los enfermos y por otro es quien diagnostica y descubre los males, por ello, es común la reticencia a acudir a ellos y consecuentemente, el escaso sentido de prevención (García y Alda, 2007).

En la cultura gitana se observan cuatro modelos de atención de salud que coexisten: el modelo tradicional, el modelo biomédico o científico, la Iglesia Evangélica o "el culto" y la auto-atención (Lagunas, 1999).

Cuando la enfermedad es leve (catarro, dolor de cabeza, etc.) se acude primeramente a la farmacia. Las mujeres son las que se ocupan de la compra y administración de fármacos. Ellas son las auténticas gestoras de salud de la familia; es la que determina la auto-atención (Otegui, 2005; Lagunas, 1999). En el caso de la fiebre, por ejemplo, la mayoría de los gitanos no están familiarizados con el uso de los termómetros y por lo tanto no los utilizan. Aunque aproximadamente la mitad de la población acude a sus médicos, la otra mitad recurre a la automedicación (paracetamol o AAS) o, más a menudo, recurren a las medicinas tradicionales populares: tomar té, a las patatas ralladas y las compresas frías, cuando se sienten febriles, y hacen hincapié en el efecto curativo de la sudoración durante los dos o tres primeros días. 
La falta de utilización de dispositivos técnicos, tales como termómetros, y la falta de familiaridad con los valores numéricos que definen la frontera entre la temperatura corporal normal y elevada, sin embargo, no significa que la población romaní adopte medidas inapropiadas en respuesta a la enfermedad (Pavlič et al, 2011).

Respecto a la utilización del modelo biomédico, se ha observado poca utilización de los servicios sanitarios entre los adultos, pero no ocurre así en los niños. En ellos es mayor la asistencia a urgencias, al igual que la tasa de hospitalización comparado con el resto de la población (Díez, Ardura, Palacín y Cardaba, 2002; Sánchez e al, 2002). Paradójicamente existe el estereotipo de la mayor resistencia de los niños de la etnia gitana a no enfermar frente a los niños de raza no gitana (Díez et al, 2002), y que a los gitanos no les interesan los problemas de salud de sus hijos (Sastre et al, 2000).

Algunos artículos señalan que los gitanos están más afectados por algunas enfermedades como la tuberculosis, asma, diabetes, anemia, difteria, deficiencias nutricionales, tabaquismo; de VHB, debido al uso de los tatuajes, y de enfermedades infecciosas en general. (Pavlič et al, 2011; Cabedo et al, 2000; Casals et al, 2012; García y Alda, 2007; Masseria et al, 2010; Kelly, 2006; Skodova et al, 2010). En cuanto al HIV-SIDA R. Otegui (2005) señala que siguen los mismos parámetros epidemiológicos que la población paya, si bien si existe una creciente preocupación acerca de la vulnerabilidad de la población gitana al HIV-SIDA y a las enfermedades de trasmisión sexual.

Son abundantes los artículos publicados que estudian alguna enfermedad genética específica de la población gitana y su relación con las elevadas tasas de consanguinidad que presentan. Se han determinado varias enfermedades causadas por la misma mutación genética recesiva, sin embargo su diagnóstico es frecuentemente un problema y son a menudo sub-diagnosticadas o no diagnosticadas correctamente. Un ejemplo son las cataratas congénitas, el dimorfismo facial y neuropatía desmielinizante, el no-síndrome de sordera prelocutiva y el síndrome miasténico congénito. (Seeman y Sisková, 2006).

Existe una recomendación desde el sistema sanitario que se refiere a la inclusión de la Mediación Intercultural para ejercer de puente entre la comunidad gitana y la sociedad mayoritaria para promover un cambio constructivo en las relaciones entre ambas. Los beneficios de la mediación intercultural repercuten tanto en el personal de los servicios sanitarios como en la población gitana usuaria de los mismos. La esperanza está en el respeto mutuo pasando previamente por el conocimiento ya que los estereotipos nos conducen a tener prejuicios, esto es a dar por hecho que los personas de un determinado colectivo van actuar de acuerdo al estereotipo que le corresponde según su grupo de pertenencia. (Gentil, 2006).

No hemos encontrado en nuestra revisión ninguna mención explícita al profesional de enfermería, es decir, ninguna publicación hace referencia a la visión que el pueblo gitano tiene de la figura de la enfermera. Dejamos este capítulo abierto para la reflexión ya que, considerando la idea de Menéndez (1994: 71-72) sobre el proceso salud/enfermedad/atención universal que opera en toda sociedad y que consta no sólo de representaciones y prácticas sino de saberes particulares para hacer frente a las angustias y sufrimientos, pensamos que es preciso la concienciación y la intervención de todos los profesionales de la salud para atender a las necesidades reales de los miembros 
de cualquier etnia, entendidas desde dentro de la propia cultura.

\section{CONCLUSIONES}

Los cuidados están polarizados sobre una sola cultura: la occidental. Además, la cultura hospitalaria es muy etnocéntrica (los diagnósticos racionales, las maneras de vivir según las enfermedades, -diabetes, infarto-...). El personal de enfermería tiene que salir del etnocentrismo y aprender a respetar y a reconocer los valores de cada cultura, de esta manera su actuación se verá fortalecida y adquirirá mayor credibilidad. Esta nueva visión se ha ido alimentando a medida que aumentaban los movimientos migratorios, a la vez que se ha reconocido el concepto de salud como multidimensional, en el que se conjugan diversos aspectos y ámbitos que van más allá de lo puramente físico.

Existen pocas publicaciones que traten este tema y ninguna que se refiera específicamente a la visión que este colectivo tiene del personal de enfermería.

La participación de los miembros de esta etnia en los programas de prevención de salud es deficitaria, comparada con la población no gitana, principalmente porque la salud no es percibida como una de las necesidades más prioritarias, y la preocupación empieza cuando aparecen los síntomas llamativos o invalidantes.

\section{BIBLIOGRAFÍA}

- Cabedo García, V.R., Ortells i Ros, E., Baquero Toledo, L., Bosch Girona, N., Montero Royo, A., Nácher Fernandez, A., et al. (2000) Cómo son y de qué padecen los gitanos. Atención Primaria, 26 (1), 21-25.

- Casabona Martínez, I., Lillo Crespo, M. (2006) Gestión de cuidados de salud y competencia cultural. Cultura de los cuidados, 20: 83-86.
- Casals, M., Pila, P., Langohr, K., Millet, J.P., Caylà, J.A. (2012) Incidence of infectious diseases and survival among the Roma population: a longitudinal cohort study. European Journal of Public Health, 22 (2), 262-266.

- Díez López, I., Ardura Fernández, J., Palacín Mínguez, E., Cardaba Arranz, M. (2002) Influencia de la etnia gitana en el ingreso hospitalario de lactantes y su patología prevalente. Anales Españoles de Pediatría, 57 (3), 215-219.

- García Campayo, J., Alda, M. (2007) Conducta de enfermedad y características culturales de la etnia gitana en España. Actas Españolas de Psiquiatria, 35 (1), 59-66.

- Gentil García, I. (2006) Enfermería y personas de otras culturas. Cultura de los cuidados, 19, 37-40.

- Kelly, J.A., Amirkhanian, Y.A., Kabakchieva, E., Vassileva, S., McAuliffe, T.L., DiFranceisco, W.J., et al.(2006) Prevention of HIV and sexually transmitted diseases in high risk social networks of young Roma (Gypsy) men in Bulgaria: randomised controlled trial. British Medical Journal, 333 (7578), 1098-1101.

- Kenrik, D. (1995) Los Gitanos: de la India al Mediterráneo. Ed. Presencia Gitana, Madrid.

- Lagunas Arias, D. (1999) Resolviendo la salud. Los gitanos catalanes. Gazeta de antropología, 15. http://www. ugr.es/ pwlac/G15_12David_Lagunas_Arias.pdf.

- Leininger, M. (1991) Culture Care Diversity \& Universality: A Theory of Nursing. National League for Nursing, (15), 2402.

- Masseria, C., Mladovsky, P., Hernández-Quevedo, C. (2010) The socio-economic determinates of the health status of Roma in comparison with non-Roma in Bulgaria, Hungary and Romania. European journal of Public Health, 20 (5), 549-54.

- Menéndez, E. (1994) La enfermedad y la curación ¿Qué es medicina tradicional? Alteridades, 4, 71-2.

- Otegui, R. (2005) "Una cosa fea”: VIH-SIDA y sistema de género entre los gitanos españoles. Revista de Antropología Social, 14, 145-172.

- Pavlič, D.R., Zelko, E., Kersnik, J., Lolić, V. (2011) Health beliefs and practices among Slovenian roma and their response to febrile illnesses: A qualitative study. Zdravstveno Varstvo, 50 (3), 169-174. 


\section{ᄃultura de las Cuidados}

- Ramírez Hita, S. (2007) Entre calles estrechas. Gitanos: prácticas y saberes médicos. Prologo de Oriol Romaní. Ediciones Bellaterra, S.L., Barcelona.

- Sanchez Serrano, F.J., Zubiaur Cantalapiedra, A., Herrero Galiana, A., Gallart Martínez, Ma․D., Jiménez Yáñez, R., Sanguino López, L., Flores Serrano, J. (2002) Diferencia étnica en la actividad asistencial en urgencias. Aproximación a la realidad gitana. Anales Españoles de Pediatría, 56, 17-22.

- San Roman, T. (1986) Entre la marginación y el racismo. Reflexiones sobre la vida de los gitanos (pp. 66-72). Alianza editorial, Madrid.

- Sastre Gussoni, E., Miranda León, M.T., Muñoz Hoyos, A., Galdó Muñoz, G. (2000) Situación de salud entre niños gitanos y no gitanos de una comarca granadina. Anales de Pediatría, 53 (3), 223-228.
- Seeman, P., SisKová, D. (2006) Autosomal Recessive Ethnical Diseases of Czech Roma Population. Casopis Lekaru Ceskych, 145 (7), 557-560.

- BOCG. Informe de la Subcomisión para el estudio de la problemática del pueblo gitano. Congreso de los Diputados serie D num.520, de 17/12/1999. (num. expediente 154/000028).

- Skodova, Z., Van Dijk, J.P., Nagyova, I., Rosenberger, J., Ondusova, D.,Studencan, M., Reijneveld, S.A. (2010) Psycosocial factors of coronary heart disease and quality of life among Roma coronary patients: a study matched by socioeconomic position. International Journal of Public Health, 55 (5), 373-80.

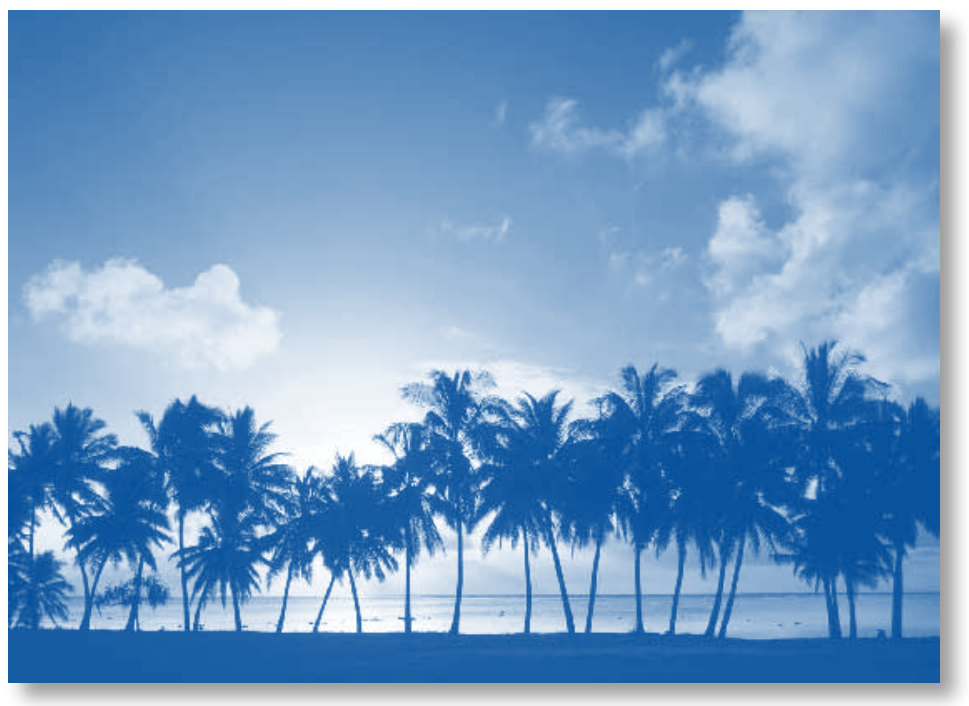

